\title{
A CHARACTERISATION OF COMPACT MINIMAL HYPERSURFACES IN A UNIT SPHERE*
}

\author{
QING-Ming Cheng
}

Dedicated to Professor Hisao Nakagawa on his 60th birthday

\begin{abstract}
In this note, we show that the totally geodesic sphere, Clifford torus and Cartan hypersurface are the only compact minimal hypersurfaces in $S^{4}(1)$ with constant scalar curvature if the Ricci curvature is not less than -1 .
\end{abstract}

Let $M$ be a compact minimal hypersurface in a unit sphere $S^{n+1}(1)$. It is wellknown that if $S \leqslant n, M$ is a totally geodesic hypersurface or Clifford torus, where $S$ is the squared norm of the second fundamental form (see Chern, do Carmo and Kobayashi [3]). Yau [8] also characterised the totally geodesic hypersurface and Clifford torus by the sectional curvature which is not less than 0 . When $n=3$, the author and Jiang [2] proved that if the Ricci curvature is not less than $1 / 2$, then $M$ is totally goedesic. Doi [5] proved that if the Ricci curvature $\operatorname{Ric}(M) \geqslant 0$ and the scalar curvature is constant, then $M$ is a totally geodesic hypersurface or Clifford torus. On the other hand, we also know that the following Cartan hypersurface has constant scalar curvature and its Ricci curvature is not less than -1 :

$$
2 x_{5}^{3}+3\left(x_{1}^{2}+x_{2}^{2}\right) x_{5}-6\left(x_{3}^{2}+x_{4}^{2}\right) x_{5}+3 \sqrt{3}\left(x_{1}^{2}-x_{2}^{2}\right) x_{4}+6 \sqrt{3} x_{1} x_{2} x_{3}=2,
$$

where $\left(x_{1}, \ldots, x_{5}\right)$ is the natural coordinate system in $\mathbb{R}^{5}$. Hence, the known examples of 3-dimensional compact minimal hypersurfaces in the unit sphere $S^{4}(1)$ with constant scalar curvature are only the totally geodesic sphere $S^{\mathbf{s}}(1)$, Clifford torus and Cartan hypersurface above. Their Ricci curvatures are not less than -1 . A natural problem is that if $M$ is a compact minimal hypersurface in $S^{4}(1)$ with constant scalar curvature and its Ricci curvature is not less than -1 , then is it one of the above minimal hypersurfaces?

In this note, we give an affirmative answer for the above problem, that is, we prove the following:

Received 5 March 1992

The project supported by NNSFC.

Copyright Clearance Centre, Inc. Serial-fee code: 0004-9729/93 \$A2.00+0.00. 
THEOREM. Let $M$ be a 3-dimensional compact minimal hypersurface in a unit sphere $S^{4}(1)$ with constant scalar curvature. If the Ricci curvature of $M$ is not less than -1 , then $M$ is totally geodesic, or a Clifford torus or a Cartan hypersurface.

Proof: For any point $p$ in $M$, we choose a local orthonormal frame field such that $h_{i j}=\lambda_{i} \delta_{i j}$, where $h_{i j}$ is the component of the second fundamental form. We consider function $f=\sum \lambda_{i}^{3}$ which does not depend on the choice of the local orthonormal frame field. If $f$ is constant, then $M$ has constant principal curvatures since $M$ is minimal and has constant scalar curvature. Hence $M$ is an isoparametric hypersurface. According to the classification of isoparametric hypersurfaces due to Cartan when $n=3$, we know that Theorem is true. Next we will prove that $f$ must be constant.

(a) If there exists a point $p$ in $M$ such that $f(p)=0$, we have, at point $p$,

$$
\begin{aligned}
& \lambda_{1}+\lambda_{2}+\lambda_{3}=0 \\
& \lambda_{1}^{3}+\lambda_{2}^{2}+\lambda_{3}^{2}=S, \\
& \lambda_{1}^{3}+\lambda_{2}^{3}+\lambda_{3}^{3}=0
\end{aligned}
$$

where $S$ is the squared norm of the second fundamental form. According to the Gaussian equation, we get that $S$ is constant since $M$ has constant scalar curvature. From (1), (2) and (3), we have $\lambda_{1}=-\sqrt{S / 2}, \lambda_{2}=0$ and $\lambda_{3}=\sqrt{S / 2}$ at point $p$. From the Gaussian equation and the assumptions of the Theorem, we obtain

$$
R_{i i}=2-\lambda_{i}^{2} \geqslant-1,
$$

where $R_{i i}$ is the component of the Ricci curvature tensor. Hence, at point $p, R_{11}=$ $2-S / 2 \geqslant-1$. Thus $S \leqslant 6$. Since $S$ is constant, we know that $S=0,3$ or 6 on $M$ from the result in [1]. Then [4, Corollary 1] due to de Almeida and Brito implies that $M$ is isoparametric. Therefore the Theorem is valid in this case.

(b) If $f(p) \neq 0$ for any point $p$ in $M$, without loss of generality, we assume $f<0$. Because $M$ is compact, we know that there exists a point $p$ in $M$ such that $f(p)=\max f<0$. Therefore, at point $p$,

$$
\begin{aligned}
& \lambda_{1}+\lambda_{2}+\lambda_{3}=0 \\
& \lambda_{1}^{2}+\lambda_{2}^{2}+\lambda_{3}^{2}=S, \\
& \lambda_{1}^{3}+\lambda_{2}^{3}+\lambda_{3}^{3}=\max f .
\end{aligned}
$$

From the following Lemma, we know that $f$ is constnat or $\lambda_{1}, \lambda_{2}$ and $\lambda_{3}$ are distinct at point $p$.

Leмma. (See Okumura [6]) Let $a_{1}, a_{2}$ and $a_{3}$ be real numbers satisfying $\sum a_{i}=$ 0 and $\sum a_{i}^{2}=k^{2}$ for $k>0$. Then

$$
\left|\sum a_{i}^{3}\right| \leqslant k^{3} / \sqrt{6} .
$$


The equality holds if and only if two of them are equal with each other.

If $f$ is constant, then the Theorem is valid. If $\lambda_{1}, \lambda_{2}$ and $\lambda_{3}$ are distinct at point $p$, from the assumptions of the Theorem and $f(p)=\max f$, we have

$$
\begin{array}{rlrl}
\sum h_{i i k}=0 & & \text { for any } k, \\
\sum \lambda_{i} h_{i i k}=0 & & \text { for any } k, \\
\sum \lambda_{i}^{2} h_{i i k}=0 & \text { for any } k,
\end{array}
$$

where $h_{i j k}$ is the component of the covariant derivative of the second fundamental form. Therefore from (4), (5) and (6), we conclude that $h_{i i k}=0$ for any $i$ and $k$ since $\lambda_{1}$, $\lambda_{2}$ and $\lambda_{3}$ are distinct. A direct and simple computation yields

$$
\Delta f=3(3-S) f+6 \sum \lambda_{i} h_{i j k}^{2},
$$

where $\Delta$ is the Laplacian on $M$. Since $f$ obtains its maximum at point $p$, we get

$$
(3-S) f+2 \sum \lambda_{i} h_{i j k}^{2} \leqslant 0 .
$$

Because $\lambda_{1}+\lambda_{2}+\lambda_{3}=0$ and $h_{i i k}=0$ for any $i$ and $k$, we have

$$
\begin{aligned}
\sum \lambda_{i} h_{i j k}^{2} & =(1 / 3) \sum\left(\lambda_{i}+\lambda_{j}+\lambda_{k}\right) h_{i j k}^{2} \\
& =(1 / 3) \sum_{i \neq j \neq k}\left(\lambda_{i}+\lambda_{j}+\lambda_{k}\right) h_{i j k}^{2}+\sum_{i \neq k}\left(2 \lambda_{i}+\lambda_{k}\right) h_{i j k}^{2}+\sum_{i} \lambda_{i} h_{i i i}^{2} \\
& =0 .
\end{aligned}
$$

Hence $(3-S) f \leqslant 0$. Thus $S \leqslant 3$ from $f(p)<0$. From the result due to Chern, do Carmo and Kobayashi [3], we obtain that $M$ is totally geodesic or a Clifford torus.

From (a) and (b), we complete the proof of the Theorem.

Making use of the generalised maximum principle due to Omori [7] and Yau [9] and the similar proof to the one of the Theorem, we can prove the following statement.

TheOREM 1. Let $M$ be a 3-dimensional complete minimal hypersurface in $S^{4}(1)$ with constant scalar curvature. If the Ricci curvature of $M$ is not less than -1 , then $S=0,3$ or 6 , where $S$ is the squared norm of the second fundamental form.

\section{REFERENCES}

[1] Q.M. Cheng, 'Complete minimal hypersurfaces in $S^{4}(1)$ with constant scalar curvature', Osaka Math. J. 27 (1990), 885-892. 
[2] Q.M. Cheng and B. Jiang, '3-dimensional submanifolds of spheres with parallel mean curvature vector', Tsukuba J. Math. 16 (1992), 321-334.

[3] S.S. Chern, M. do Carmo and S. Kobayashi, 'Minimal submanifolds of a sphere with second fundamental form of constant length', in Functional analysis and related fields (Springer-Verlag, Berlin, Heidelberg, New York, 1970), pp. 59-75.

[4] S. de Almeida and F.G. Brito, 'Closed 3-dimensional hypersurfaces with constant mean curvature and constant scalar curvature', Duke Math. J. 61 (1990), 195-206.

[5] N. Doi, 'On compact minimal hypersurfaces in a sphere with constant scalar curvature', Nagoya Math. J. 78 (1980), 177-188.

[6] M. Okumura, 'Hypersurfaces and a pinching problem on the second fundamental tensor', Amer. J. Math. 96 (1974), 207-213.

[7] H. Omori, 'Isometric immersions of Riemannian manifolds', J. Math. Soc. Japan 19 (1967), 205-214.

[8] S.T. Yau, 'Submanifolds with constant mean curvature II', Amer. J. Math. 97 (1975), 76-100.

[9] S.T. Yau, 'Hamonic functions on complete Riemannian manifolds', Comm. Pure Appl. Math. 28 (1975), 201-228.

Institute of Mathematics

Fudan University

Shanghai 200433

Peoples Republic of Chins 